\title{
Voice Assisted Embedded Navigation System for the Visually Impaired
}

\author{
V. Ramya \\ Assistant Professor \\ Department of Computer \\ Science and Engineering, \\ Annamalai University, \\ Chidambaram,Tamilnadu
}

\author{
Laxmi Raja \\ Research Scholar \\ Department of Computer \\ Science and Engineering, \\ Annamalai University, \\ Chidambaram,Tamilnadu
}

\author{
B Palaniappan \\ Professor \\ Department of Computer \\ Science and Engineering, \\ Annamalai University, \\ Chidambaram,Tamilnadu
}

\begin{abstract}
For visually impaired people, a cane is a close companion helping them to detect and avoid obstacles while walking. But while walking in a new or changed environment, it is hard for them to guess where they are. Also it will be a problem when they lose memory of locations and places. The standard method for taking notes by visually impaired is to emboss Braille dots on a paper, which can be read by feeling the dots by fingers [16]. It is difficult to imprint, copy, transfer or edit these writings. With the advancement in modern day electronic sensors, touch sensing and microcontroller technology, the proposed system aids the visually impaired in navigation via audible messages and haptic feedback, helping them localize where they are and to improve their mobility. This system supports the visually impaired to enter notes and control device operation via touch keypad. The device also provides user information in audio format, including navigation direction, ambient light and temperature condition. The aim of this project is to help visually impaired to improve their communication and provides independency during walking in even unknown areas.
\end{abstract}

\section{Keywords}

ARM, MEMS, Touch Keypad, and Voice assisted Navigation.

\section{INTRODUCTION}

Worldwide, there are 314 million visually impaired and 45 million of them are blind. About $87 \%$ of the visually impaired live in the developing countries, over them 15 million people are from India [19]. Blind and Visual impaired people face several accessibility and mobility problems in day to day life. Some tasks or situations have physical constraints, sometimes impossible for them to overcome [16]. Those difficulties are related with a lack of autonomy or information that could help them to avoid obstacles. These problems can be overcome by improving the human ability for sensing and recognizing it [12]. In general, urban accidents are influenced by a lack of right signalization or by a chance, and some related causes like public phone booths, mailboxes, poles, dump-carts and twigs of trees.

Moving through an unknown environment becomes a real challenge when we can't rely on our own eyes [17]. Since dynamic obstacles usually produce noise while moving, blind people develop their sense of hearing to localize them [18]. However they are reduced to their sense of touch when the matter is to determine where an inanimate object exactly is. The common way for navigating of visionless person is using a walking cane. The walking cane is a simple and purely mechanical device dedicated to detect static obstacles on the ground, uneven surfaces, holes and steps via simple tactileforce feedback. This device is light, portable, but range limited to its own size and it is not usable for dynamic obstacles detection.

Many commercial devices are available in the market for navigation for the blind. But lack of user-friendliness makes them less useful for the consumers. Thus our system is designed with ARM processor as it heart which can give the following applications; the system gives an audio output through microphone to the user about the direction in which the user is travelling. The system also provides a light source providing ambient light. The purpose of this light is to announce the presence of the user during low light times like night hours. Temperature of the environment can be sensed and output sent as voice. The device can take notes using a touch keypad when needed. It also has a MP3 player to play the user's favorite music when needed and a SD card for storage.

\section{RELATED WORK}

Katherine J. et.al [1] has developed HALO which gives visually impaired the freedom to travel independently in unknown environments, but they cannot warn the user of overhead hazards such as tree branches. HALO provides haptic cues to warn a visually impaired user of low-hanging obstacles during white cane navigation. The Haptic Alerts for Low-hanging Obstacles (HALO) system is a portable and affordable attachment to traditional white canes. By pairing distance data acquired from an ultrasonic range sensor with vibration feedback delivered by an eccentric mass motor, the device aims to alert users of low-hanging obstacles without interfering with the standard functionality of a white cane.

Esteban Bayro Kaiser et.al [2] have developed "Wearable navigation system for the blind" that will map and track the position of the pedestrian during the exploration of the unknown environment. In order to build this system, the well known Simultaneous Localization and Mapping (SLAM) from mobile robotics will be implemented. Once a map is created the user can be guided efficiently by a route selecting method. The user will be equipped with a short range Laser, an inertial measurement unit (IMU), a wearable computer for data processing and an audio bone headphone. This system does not intent to replace the use of the white cane. However, the purpose is to gather contextual information to aid the user in navigating with the white cane.

S.Kammouna et.al [3] have designed the NAVIG device which aims to complement conventional mobility aids (i.e. 
white cane and guide dog), while also adding unique features to localize specific objects in the environment, restore some visuomotor abilities and assist navigation. The NAVIG project (Navigation Assisted by embedded Vision and GNSS, 2009-2012)1 was created in an attempt to address some of these issues. The aim was to design and evaluate a powerful assistive device combining both micro (sensing the immediate environment) and macro navigation (reaching a remote destination) functions. The micro navigation functions serve to restore a set of sensory motor behaviors based on visual objects localization. The macro navigation functions provide the user with global orientation and navigation skills. This paper is an overview of the project that roughly describes the main work packages with their specific objectives, indicating pointers to detailed publications where additional details can be found.

Sin Murad et.al [4] has developed RFAIDE - An RFID Based Navigation and Object Recognition Assistant for Visually Impaired People. This is a system utilizing Radio Frequency Identification (RFID) for the assistance of blind people. The system incorporates a mobile RFID reader module with an integrated ZigBee transceiver for transmitting the tag's information. Utensils and other objects in the house or building are embedded with passive RFID tags (transponders) along with an audio file, recorded for and unique to each object, residing on the server. This system further takes in the way finding technique by employing an RFID tag grid using an ample separation area. The reader reads the tags and transmits the data wirelessly to the server PC which in turn scans for the particular ID in the database and plays the corresponding audio file. A self designed coordinates system with a server side routing application is used for routing the person to a particular room requested, based on his current tag coordinates. The audio playback is relayed wirelessly using an FM transmitter to either a headset with FM receiver or a Smart Phone's FM radio.

A.R.García et.al [5] has developed Electronic long cane for locomotion. This is an assistive technology device called ELC (Electronic Long Cane) which has an ergonomic design along with an embedded electronics inside the grip of a traditional long cane, which, by mean of haptics, spreads human spatial and tactile perception. The device signalizes obstacles above waistline and warns about potential collisions. Qualitative evaluation of an ELC prototype was carried out by voluntary blind people. The obtained results showed the ELC effectiveness for detecting physical barriers located above of the imaginary waistline, so, contributing to a better perception of the surrounding space by blind people.

\section{SYSTEM DESIGN}

\subsection{Voice Based Navigation System}

The system uses ARM Cortex-M3 as Microcontroller. Users are assisted through voice messages using Voice Codec and Head phone. Whenever temperature is asked by user, it is sensed using LM35 Temperature sensor. Light is sensed using LDR. If light sensor detects dark, LED switches on. Touch Capacitive Sensor is used to control the system. Direction in which the user is travelling is computed using MEMS and DC-Servo Motor and a voice output related to direction is received by the user. All data are stored in a 2-GB Memory SD-Card.

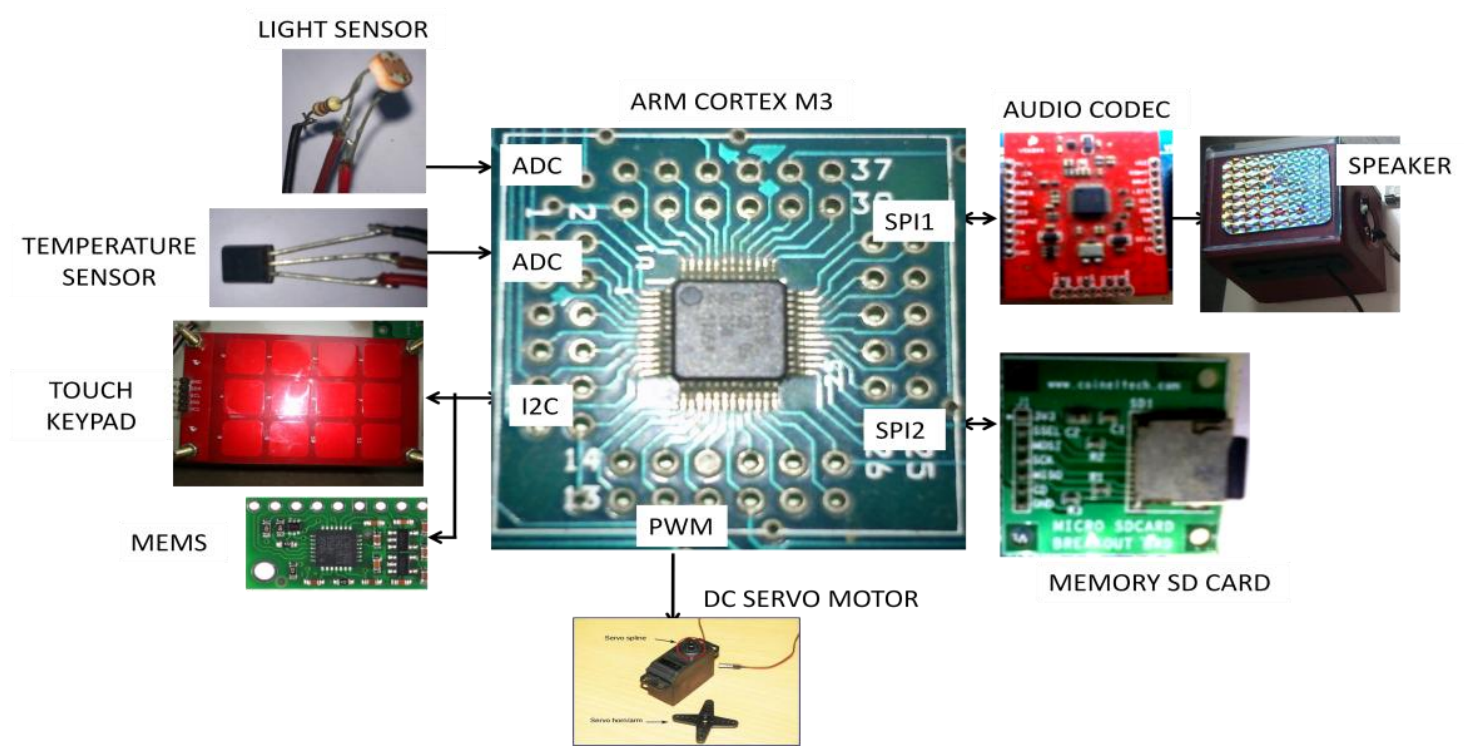

Figure 1: Voice based Navigation system

\section{HARDWARE DESCRIPTION \\ 4.1 Microcontroller}

System-on-chip solutions based on ARM embedded processors address many different market segments including enterprise applications, automotive systems, home networking and wireless technologies. The ARM Cortex family of processors provides a standard architecture to address the broad performance spectrum required by these diverse technologies. The ARM Cortex family includes processors based on the three distinct profiles of the ARMv7 architecture; the A profile for sophisticated, high-end applications running open and complex operating systems; the R profile for realtime systems; and the $M$ profile optimized for cost-sensitive and microcontroller applications. The Cortex-M3 processor is the first ARM processor based on the ARMv7-M architecture and has been specifically designed to achieve high system performance in power and cost-sensitive embedded 
applications, such as microcontrollers, automotive body systems, industrial control systems and wireless networking, while significantly simplifying programmability to make the ARM architecture an option for even the simplest application.

\subsection{Magnetometer \& Accelerometer}

Magnetometer and Accelerometer (MEMS) is used to detect the direction in which the user is travelling. MEMS is connected through I2C interface to the ARM Processor. The output of the MEMS is processed in microcontroller to produce voice output in degrees. It thus helps the user to know whether he is travelling in the proper direction. LSM303DLH is the MEMS being used in the system. It has 28 pins. SCL_M and SDA_M are used for magnetic signal interface I2C serial clock and data. Similarly, SCL_A and SDA_A are used for linear acceleration signal interface I2C serial clock and data. Fig 2 and 3 shows the image of MEMS and its interfacing with ARM Cortex

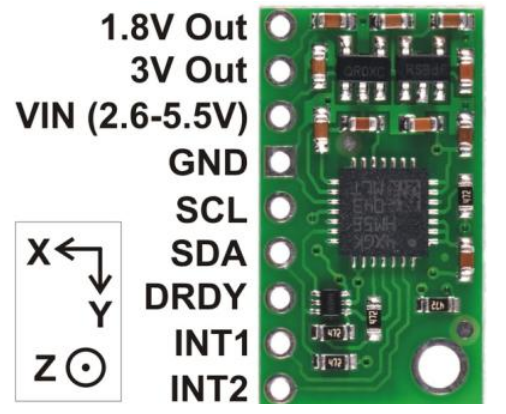

Figure 2: Image of Magnetometer \& Accelerometer

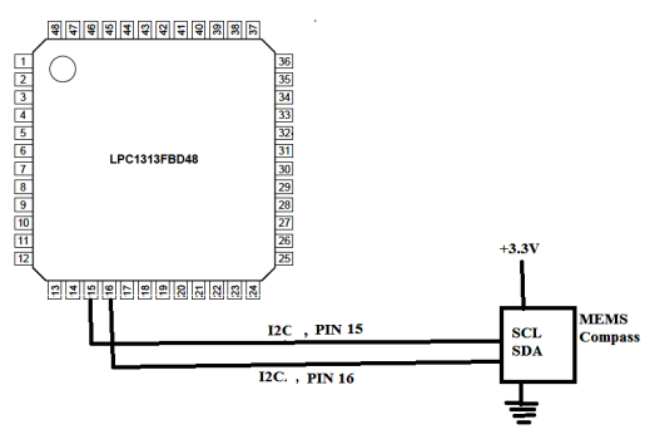

Figure 3: MEMS interfacing with ARM Cortex

\subsection{Capacitive Touch Sensor}

Capacitive Touch Sensor is the next important module in the system after ARM Processor as it is required to control all the commands in the system. For inserting any instruction or notes, the user needs the Touch Keypad. MPR121 is the capacitive touch sensor being used in our system. It contains 12 electrodes. MPR121 is interfaced to ARM through I2C port. Figure 4 shows image of touch sensor.

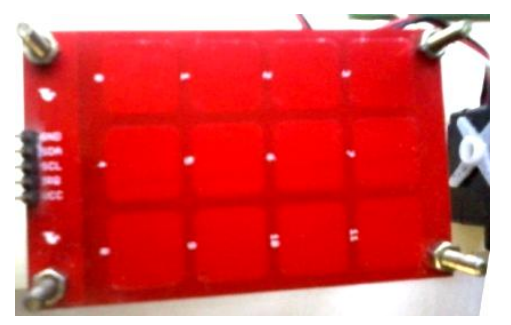

Figure 4: Image of Capacitive Touch Sensor

\subsection{Audio CODEC}

The system assists the user through voice messages. This feature is only possible due to audio codec. Audio codec is responsible to convert MP3 files to voice form. Pre recorded audio MP3 files are stored in the memory SD card. Whenever the microcontroller calls a particular MP3 file, it is processed and played using audio codec. We can listen to the output of audio codec through a speaker or headphone. The system uses VS1053b audio codec. It contains 48 pins. VS1053b is a single-chip MP3 audio decoder. Figure 5 shows image of VS1053b and Figure 6 shows CODEC interfacing with ARM Cortex.

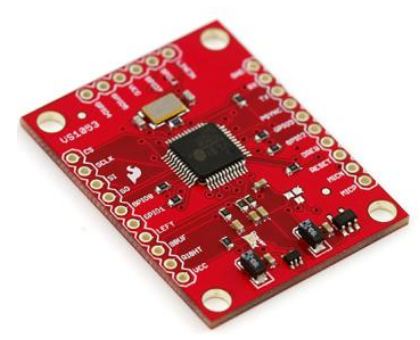

Figure 5: Image of Audio CODEC

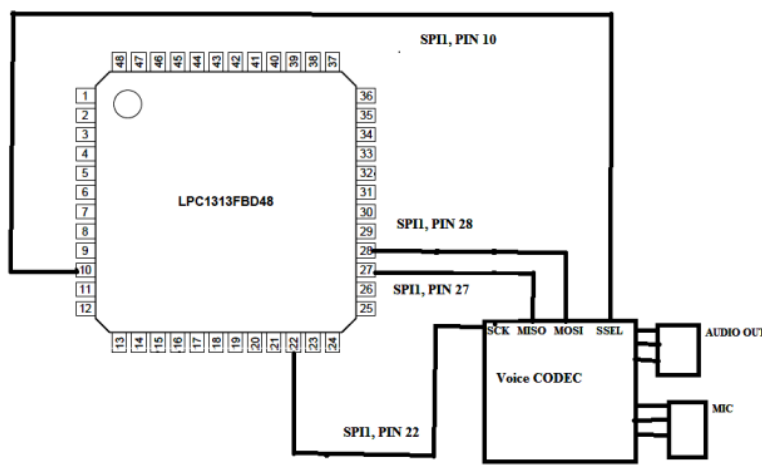

Figure 6: Audio CODEC interfacing with ARM Cortex

\subsection{Memory SD card}

The Memory SD card is a storage device but unlike other storage devices it is small in size and like Pen drives a standard port to insert SD card is universally same.

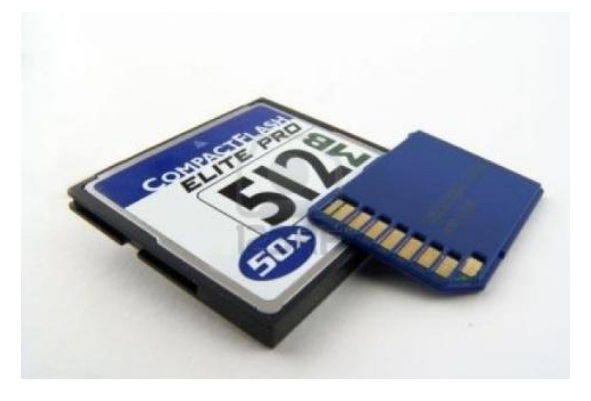

Figure 7: Image of Memory card and its holder So any SD card can be used in any SD card holder. In this device, the user needs to store lot of information like prerecorded audio warnings and MP3 music. Figure 7 show image of Memory card and its holder. 


\subsection{Servo}

DC Servo is connected PWM pin of microcontroller. Here, pin PI01 9 is interfaced with DC Servo. The Servo Motors come with three wires or leads. Two of these wires are to provide ground and positive supply to the servo DC motor. The third wire is for the control signal. These wires of a servo motor are color coded. The red wire is the DC supply lead and must be connected to a DC voltage supply in the range of $4.8 \mathrm{~V}$ to $6 \mathrm{~V}$. The black wire is to provide ground. Spline is the place where a servo arm is connected. It is analogous to the shaft of a common DC motor.

Unlike DC motors, reversing the ground and positive supply connections does not change the direction (of rotation) of a servo. This may, in fact, damage the servo motor. That is why it is important to properly account for the order of wires in a servo motor. Figure 8 shows prototype of DC servo motor

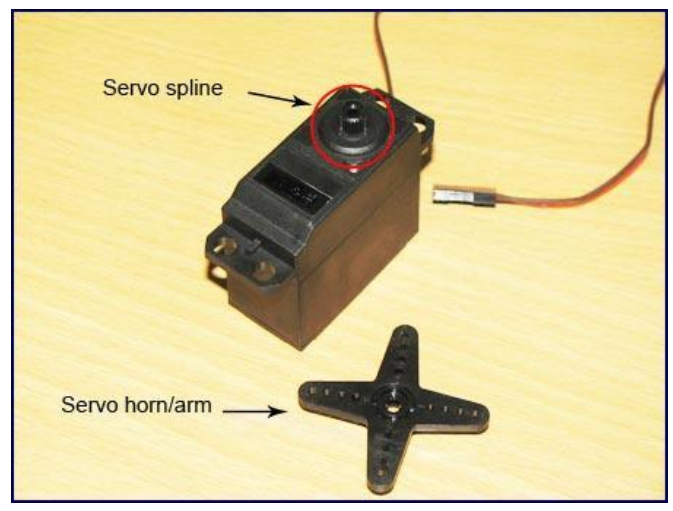

Figure 8: Prototype of DC Servo

\subsubsection{Servo Control:}

The servo motor can be moved to a desired angular position by sending PWM (pulse width modulated) signals on the control wire. The servo understands the language of pulse position modulation. A pulse of width varying from 1 millisecond to 2 milliseconds in a repeated time frame is sent to the servo for around 50 times in a second. The width of the pulse determines the angular position. For example, a pulse of 1 millisecond moves the servo towards $0^{\circ}$, while a 2 milliseconds wide pulse would take it to $180^{\circ}$. The pulse width for in between angular positions can be interpolated accordingly. Thus a pulse of width 1.5 milliseconds will shift the servo to $90^{\circ}$.

A sequence of such pulses (50 in one second) is required to be passed to the servo to sustain a particular angular position. When the servo receives a pulse, it can retain the corresponding angular position for next 20 milliseconds. So a pulse in every 20 millisecond time frame must be fed to the servo. Figure 9 shows the Changing of rotation by pulse width modulation in a Servo motor.

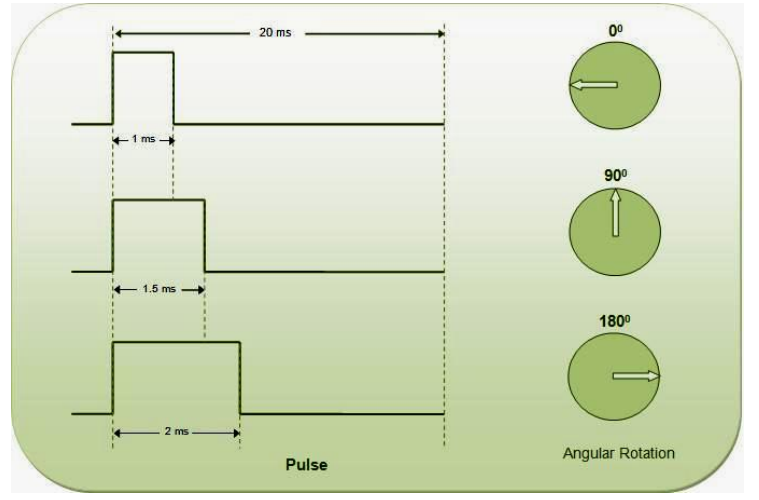

Figure 9: Changing of rotation by pulse width modulation

\subsection{Light Sensor}

Light sensor used in the system is N5AC 501085 LDR photocell. It detects light intensity and passes the information digitally. It is connected to one of the ADC pins of microcontroller. LDR photocell is needed for the device to detect the low light conditions such as darkness and the information should be relayed to the user as audio warning. Also in case of darkness, a LED light is switched on for user's assistance in dark. Figure 10 shows image of light sensor.

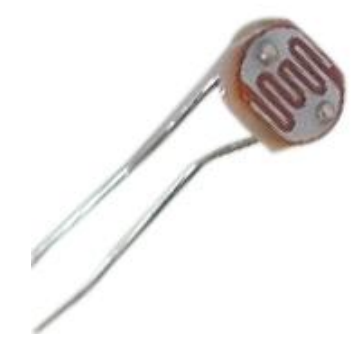

Figure 10: Prototype of Light sensor

\subsection{Temperature Sensor}

Temperature sensor used in the system is LM35. It detects temperature and passes the information digitally. It is connected to one of the ADC pins of microcontroller. Figure 11 shows prototype of temperature sensor and 12 shows its interface with the microcontroller.

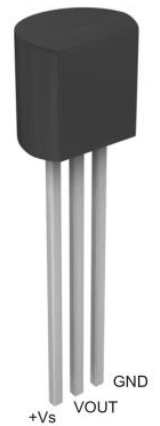

Figure 11: Prototype of Temperature sensor 


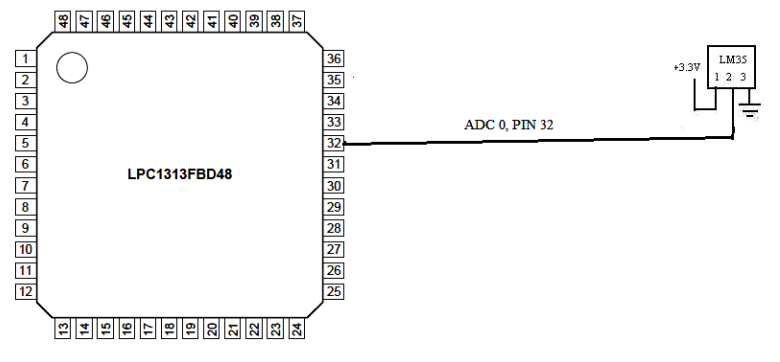

Figure 12: Temperature sensor interfacing with ARM

Cortex (M3)

\section{ARM CORTEX-M3 (LPC1313) \\ 5.1 ARM Cortex-M3}

The ARM Cortex family of processors provides a standard architecture to address the broad performance spectrum required by diverse technologies. The ARM Cortex family includes processors based on the three distinct profiles of the ARMv7 architecture; the A profile for sophisticated, high-end applications running open and complex operating systems; the $\mathrm{R}$ profile for real-time systems; and the $\mathrm{M}$ profile optimized for cost-sensitive and microcontroller applications. The Cortex-M3 processor is the first ARM processor based on the ARMv7-M architecture and has been specifically designed to achieve high system performance in power- and cost-sensitive embedded applications, such as microcontrollers, automotive body systems, industrial control systems and wireless networking, while significantly simplifying programmability to make the ARM architecture an option for even the simplest applications.

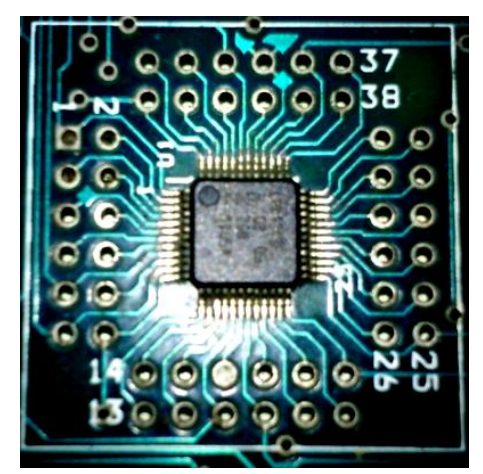

Figure 13: ARM Cortex-M3

The ARM Cortex M-3 processor integrates the central processor core, called the CM3Core, with advanced system peripherals to enable integrated capabilities like interrupt control, memory protection and system debug and trace. These peripherals are highly configurable to allow the Cortex-M3 processor to address a wide range of applications and be more closely aligned with the system requirements. The Cortex-M3 core and the integrated components have been specifically designed to meet the requirements of minimal memory implementation, reduced pin count and low power consumption. Figure 13 and 14 shows the ARM cortex M3 and its pin diagram.

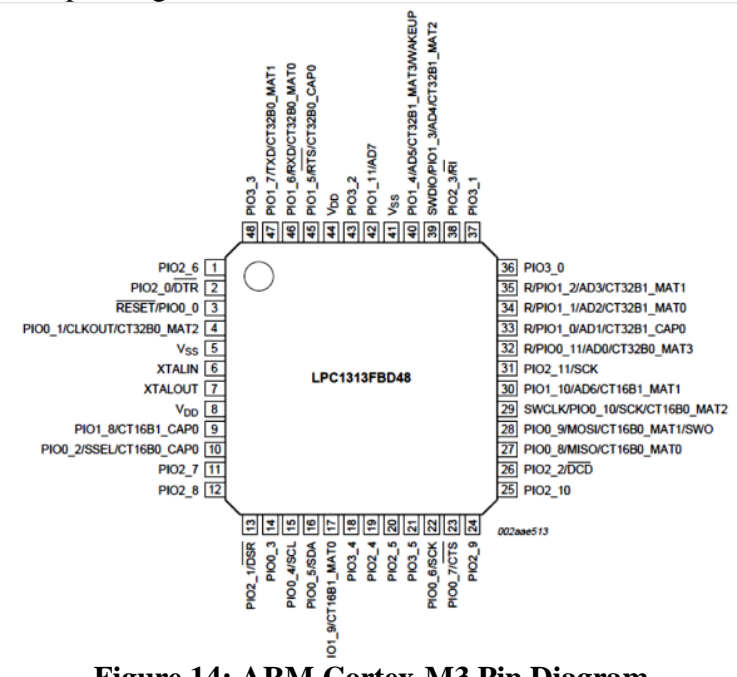

Figure 14: ARM Cortex-M3 Pin Diagram

\section{SOFTWARE DESCRIPTION}

\subsection{Embedded C}

Looking around, we find ourselves to be surrounded by various types of embedded systems. Be it a digital camera or a mobile phone or a washing machine, all of them has some kind of processor functioning inside it. Associated with each processor is the embedded software. If hardware forms the body of an embedded system, embedded processor acts as the brain, and embedded software forms its soul. It is the embedded software which primarily governs the functioning of embedded systems. Embedded systems programming is different from developing applications on a desktop computers.

\subsection{LPCXpresso IDE}

The LPC1311/13/42/43 are ARM Cortex-M3 based microcontrollers for embedded applications featuring a high level of integration and low power consumption. The ARM Cortex-M3 is a next generation core that offers system enhancements such as enhanced debug features and a higher level of support block integration. The LPC1311/13/42/43 operate at CPU frequencies of up to $72 \mathrm{MHz}$. The ARM Cortex-M3 CPU incorporates a 3-stage pipeline and uses Harvard architecture with separate local instruction and data buses as well as a third bus for peripherals. The ARM CortexM3 CPU also includes an internal prefetch unit that supports speculative branching. The peripheral complement of the LPC1311/13/42/43 includes up to $32 \mathrm{kB}$ of flash memory, up to $8 \mathrm{kB}$ of data memory, USB Device (LPC1342/43 only), one Fast-mode Plus I2C-bus interface, one UART, four general purpose timers, and up to 42 general purpose I/O pins. 


\section{RESULTS AND DISCUSSION}

The completed system is given in the Fig. 17.

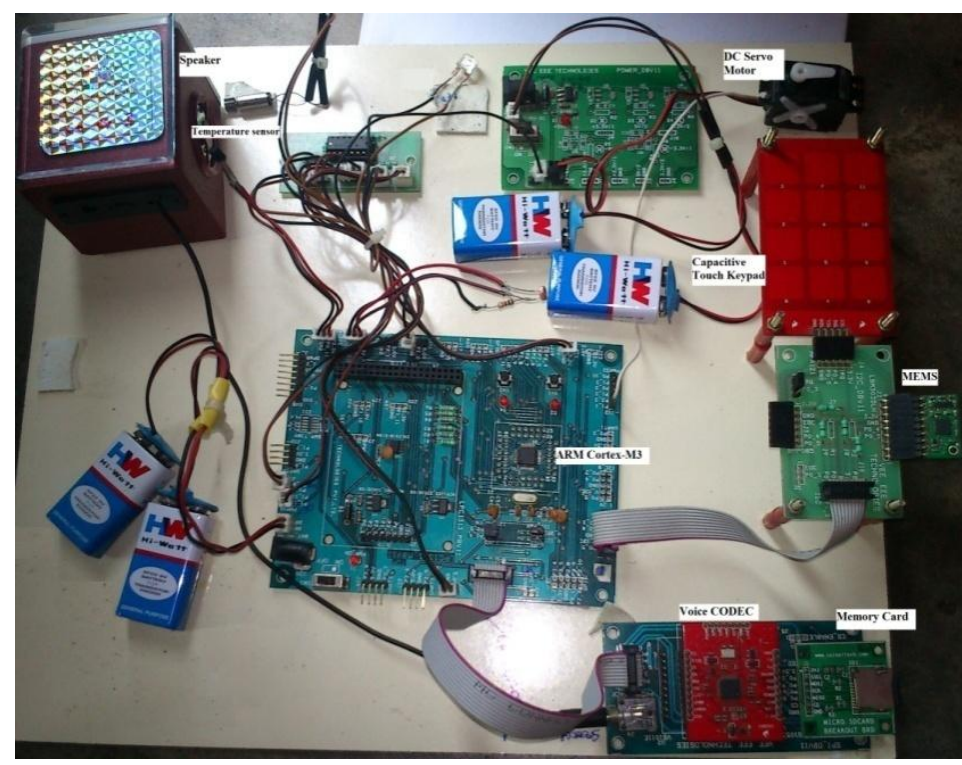

Figure 17: Prototype of Navigation device for visually impaired.

The Flow chart of the system is given in Fig. 18
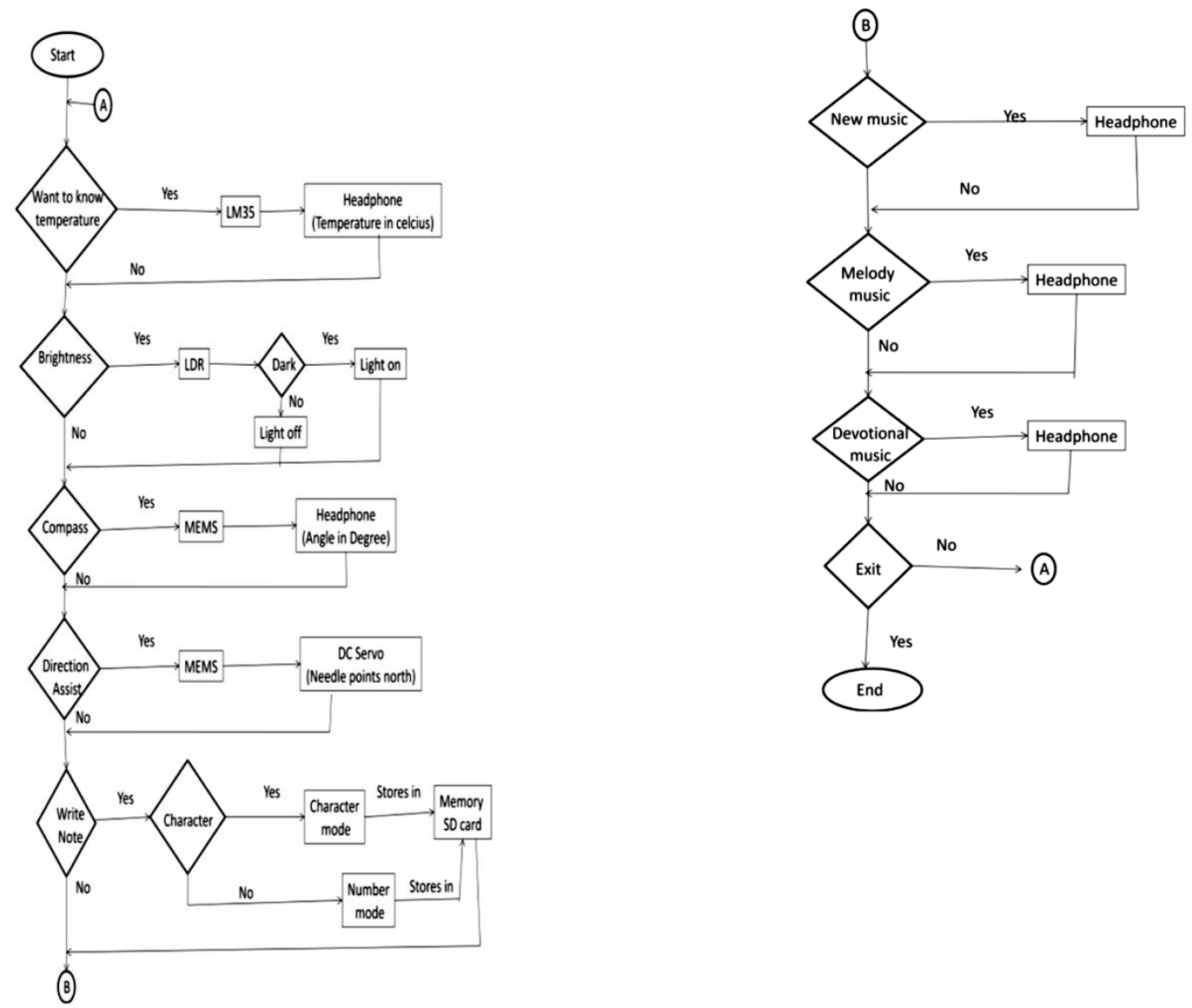

Figure 18: Flowchart of the system. 


\subsection{Taking Notes}

When the user is walking in a new area, he inputs notes regarding the area in his touch screen key pad. These notes are saved in the memory card for future use.

\subsection{Giving Commands to the System}

User gives commands in the keypad and it is decoded. The corresponding sensory information is outputted via audible messages using a head phone.

\subsection{Temperature System}

Temperature sensor senses the surrounding temperature and when the user gives command about it, the audio output announces the environmental temperature in degree Celsius.

\subsection{Ambient Light}

System will announce whether it is light or dark and if it is dark, the lights will automatically turn on, so that vehicles or bystander are cautioned about the user's proximity and location.

\subsection{Assisting navigation}

Magnetometer is a device which functions as compass. The DC Servo motor and the MEMS can be utilized by the user by feeling the direction of needle in the device. This helps the user going in correct direction.

\subsection{Entertainment}

Pre recorded MP3 audio files are stored in a FAT-32 formatted Memory SD card for the entertainment of user.

\section{CONCLUSION}

The Scope of this project is to device a system that will give more independence to the visually impaired in terms of their navigational ability in unknown areas and to improve their comfort and safety during when walking without any help such as human Guides or Guide dogs. The system has ARM processor at its heart to aide in navigation for the visually impaired. Here, ARM processor is installed with LPXpresso software. Also the temperature sensor, magnetometer and accelerometer and capacitive touch keypad are installed and working perfectly. Audio CODEC and speakers are also fitted. Using this now the user can take notes using the touch pad screen when walking in unknown areas. The user will be informed on the conditions of the surrounding temperature conditions via a prerecorded audio message via the speaker. Also the user can listen to his/her preferred music. The MEMS and DC Servo will sense the direction and inform the user whether he is walking in right direction or not. Light sensors are used to detect whether it is dark or bright. If environment is dark, light is automatically switched on announcing the presence of the user to others.

\section{REFERENCES}

[1] Katherine J. Kuchenbecker, "HALO: Haptic Alerts for Low-hanging Obstacles", White Cane Navigation Departmental Papers (MEAM), 2012.

[2] Esteban Bayro Kaiser, Michael Lawo,"Wearable Navigation System for the Visually Impaired and Blind People”, IEEE, 2012.

[3] S. Kammouna, G. Parseihian, O. Gutierrez, "Navigation and space perception assistance for the visually impairedThe NAVIG project”, IRBM Vol.33, 2012.
[4] Sin Murad, Abdullah Rehman, Arif Ali Shah, "RFAIDE - An RFID Based Navigation and Object Recognition Assistant for Visually Impaired People", IEEE, 2011.

[5] A. R. García, R. Fonseca. A. Durán, "Electronic long cane for locomotion improving on visual impaired people. A case study", IEEE, 2011

[6] Mohd Helmy Abd Wahab, Amirul A. Talib, A. Kadir, Ayob Johari, A.Noraziah, Roslina M. Sidek, Ariffin A Mutalib, "Smart cane: Assistive cane for the visually impaired people", International Journal of Computer Science Issues, Vol. 8, Issue 4, No 2, July 2011.

[7] Mihai Emanuel Basch, Robert Istvan Lorincz, David George Cristea, "A Bio-inspired Collision Avoidance System Concept for People with Visual Disabilities", International Journal Of Systems Applications Engineering \& Development, Issue 6, Volume 5, 2011.

[8] Kenneth Chee, Xyrus Ferriol, Edmund Ing, Jeffrey Leung, William Yiu, "Radar for the Blind", California Polytechnic College of Engineering, Issue 2,Vol 8, 2011.

[9] R. Fonseca Livramento da Silva, L. S. Gómez, "Design of product integrated to the urban project: evaluation of the assistive technology design and its contribution for the inclusion of visual impaired people in open urban spaces," $10^{\circ}$ Ergodesign congress internacional de ergonomia e usabilidade de interfaces humanotecnologia, pp. 195, 2010.

[10] S. Sai Santhosh, T. Sasiprabha, R. Jeberson, "BLI NAV Embedded Navigation System for Blind People", IEEE, 2010.

[11] H.Fernandes, P.Costa, V.Filipe, L.Hadjileontiadis, J.Barroso, "Stereo Vision In Blind Navigation Assistance", World Automation Congress, pp.1-6, 2010.

[12] P.Jae-Han, B.Seung-Ho, B.Moon-Hong, “An intelligent navigation method for services robots in the Smart Environment," International Conference on Control, Automation and Systems, pp. 494, 2007.

[13] Sylvain Cardin, Daniel Thalmann, Frédéric Vexo, "Wearable System for Mobility Improvement of Visually Impaired People", The Visual Computer, Vol 23, no. 2, pp. 109-118, 2007.

[14]Andreas Hub Tim Hartter Thomas Ertl., "Interactive Tracking of Movable Objects for the Blind on the Basis of Environment Models and Perception-Oriented Object Recognition Methods", ASSETS'06, 2006.

[15] Tsung-Hsiang Chang, Chien-Ju Ho, David Chawei Hsu, "iCane - A Partner for the Visually Impaired", International Conference on Embedded and Ubiquitous Computing, pp. 393-402, 2005.

[16] M. Dischinger, "Designing for all senses: accessible spaces for visually impaired citizens", Department of Space and Process School of Architecture, Chalmers University of Technology, 2000.

[17] M.A. Espinosa, S. Ungar, E. Ochaíta, Blades, "Comparing Methods for Introducing Blind and Visually Impaired People to Unfamiliar Urban Environments", Journal of Environmental Psychology, pages 277-287, 1998.

[18] F.Schmidt, "Fundamentals of Sensory Physiology", Springer, 1979.

[19] http://timesofindia.indiatimes.com/india/India-haslargest-blind-population/articleshow/2447603.cms 